\title{
Questions d'acquisition à l'interface syntaxe I sémantique
}

\author{
Hamida Demirdache \\ Université de Nantes, LLING 3827 \\ hamida.demirdache@, univ-nantes.fr
}

\begin{abstract}
Nous discuterons une des problématiques centrales qui anime aujourd'hui les recherches menées en acquisition syntaxique : celle de l'interface syntaxe / sémantique. Les questions très générales posées sont, d'une part, celle des rapports entre acquisition d'une compétence syntaxique et acquisition d'une compétence sémantique et, d'autre part, celle de comment concevoir l'acquisition à cette interface dans des modèles linguistiques qui supposent une correspondance étroite ('mapping') entre structures syntaxiques et structures sémantiques (ou Formes Logiques). Nous chercherons à illustrer concrètement les enjeux - théoriques et de méthodologie expérimentale - que soulève l'acquisition à l'interface syntaxe / sémantique à partir de travaux récents menés sur l'acquisition de la syntaxe des interrogatives.
\end{abstract}

